\title{
Inter-domain resource optimization method of software-defined multi-domain optical network
}

\author{
Zikang $\mathrm{Li}^{1,2}$, Changming Yang ${ }^{1,2}$, Zhenrong Zhang ${ }^{1,2^{*}}$ and Sifeng $\mathrm{Hu}^{1,2}$
}

\begin{abstract}
In practical application scenarios, the division of different operators or the same operator in different regions constitutes a natural network region, forming a multi-domain optical network. At the beginning, this paper introduces the hierarchical architecture of software-defined optical network (SDON) and the functional model of control plane. Then, it describes a control system of software-defined multi-domain optical network based on hierarchical control mode and briefly analyzes their basic functional model. In the end, around the problems of resource allocation between scenarios in multi-domain optical network, this paper respectively analyzes the optimization of routing resources in inter-domain and the mechanism of spectrum defragmentation. At the same time, it separately simulates the resource optimization algorithms of single control architecture and hierarchical control architecture to verify the superiority of hierarchical control system.
\end{abstract}

Keywords: Multi-domain optical network, SDON, Hierarchical architecture, Inter-domain, Resource optimization algorithm

\section{Introduction}

With the increasing demand for communication capacity, the transmission speed, communication quality, and optical network have been developed rapidly in recent years [1-3]. Optical transport went through the developments from synchronous digital hierarchy (SDH) to wavelength-division multiplexing (WDM) [4] and optical orthogonal frequency division multiplexing (O-OFDM) [5]. Meanwhile, optical networking construct technology went through the evolvements from point to point network, static wavelength routing network, Automatic Switching Optical Network (ASON) and optical network based on path computation element (PCE) [6-8]. In 2011, software-defined network (SDN) represented by OpenFlow (a network protocol) was first proposed in computer network and gradually extended to optical network $[9,10]$.

\footnotetext{
* Correspondence: zzr76@gxu.edu.cn

${ }^{1}$ School of Computer, Electronics and Information, Guangxi University, Nanning 530004, People's Republic of China

${ }^{2}$ Guangxi Key Laboratory of Multimedia Communications and Network Technology, Nanning 530004, People's Republic of China
}

By allowing the control plane to be decoupled from the data plane, software-defined optical network (SDON) brings up a new paradigm. It enables remote controllers to configure the network equipment from different hardware vendors [11]. SDON combines the advantages of optimizing fragmentation of specific networks in optical layer and the great bandwidth providing various services, and it also provides a unified control platform for different networks [12].

In practical application scenarios, the division of different operators or the same operator in different regions constitutes a natural network region, forming a multidomain optical network. With multiple domains coexisting, the administrators in each domain want an independent SDON controller to take full control of their domains [13, 14]. When confronting the cross-domain service in the whole network, how to coordinate multiple domains for collaborative controlling and allocation of routing and spectrum resources is very important. We urgently hope that a control mechanism based on these single-domain controllers can realize the control of cross-domain service and optimization of multidomain global network resources $[15,16]$. 


\section{Methods/experimental}

The aim of this paper is to solve the problem of resource allocation among domains in the multi-domain optical network scenario and improve the utilization rate of resources.

Firstly, we introduced a control system of softwaredefined multi-domain optical network based on hierarchical control mode and briefly analyzed its basic functional model. Then, we analyzed the optimization of routing resources in inter-domain and the mechanism of spectrum defragmentation. At last, we simulated the resource optimization algorithms of single control architecture and hierarchical control architecture to verify the superiority of hierarchical control system.

\section{The hierarchical control system of software- defined multi-domain optical network}

This section mainly introduces the architecture and interfaces of SDON from concepts and functions, expounds two multi-domain control scheme and the hierarchical control system structure.

\subsection{The architecture and interfaces of SDON}

Figure 1 illustrates the hierarchical structure of SDON. This architecture is divided into four planes: data plane, controller plane, application plane, and management plane [17]. The interface between the data plane and the controller plane is the data plane control interface (D-CPI). The interface between the controller plane and the application plane is the application plane control interface (A-CPI). Agent supports the concept of sharing or virtualization of basic resources. A single network element or SDN controller can have multiple agents simultaneously, but only one coordinator per network element or SDN controller, because there is only one logical management interface. The coordinator is a data plane including one or more network elements, and each network element contains a set of data forwarding or service processing resources. The control plane adopts the responsible for installing customer-specific resources and policies received from the management system mechanism of a centralized controller. The controller is equipped with basic function modules to realize basic functions such as building roads and demolition roads, and also to install corresponding specific function modules according to different application requirements. The application plane includes one or more applications. Web applications can be developed by any vendor such as equipment producers, carriers, or third-party vendors. The management plane manages the data plane, the controller plane, and the application plane through the management interface which realizes the functions of configuration, performance, alarm, billing, and so on.

The interfaces of SDON include the northbound interface and southbound interface. The northbound interface of SDON is an interface between the controller and the upper application. The upper level service applications can conveniently call the underlying network resources through an open interface. The basic functions that should be supported by the north interface include network topology acquisition, business request and distribution, connection control, channel computing, virtual network services, and

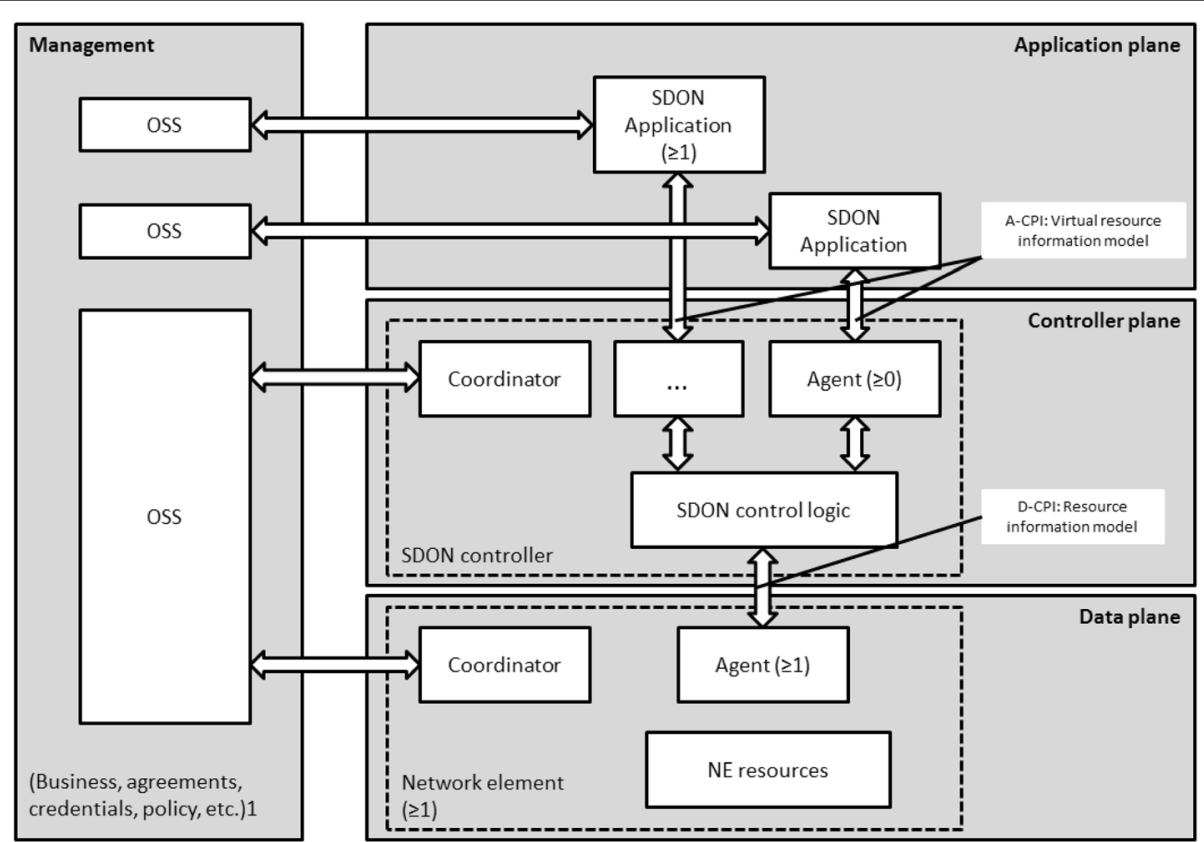

Fig. 1 The hierarchical structure of SDON. This architecture includes four planes: data plane, controller plane, application plane and management plane 
so on. The northbound interface of SDON information model should conform to the modeling method of Open Networking Foundation (ONF) general information model, as well as the provisions of ONF Transport API (application programming interface). The north interface uses Restful (representational state transfer) and SOAP (Simple Object Access Protocols) as the protocol interaction between the controller and the APP (application).

The controller south interface is an interface between the controller and the forwarding device. The controller can get the topology and resource information of the local network through the south interface to complete the local network connection and business related control functions. At the same time, the controller can get the alarm information and performance information of the local network through the south interface. At present, there are many southbound interface protocols, such as PCEP, SNMP (simple network management protocol), OFCONFIG (OpenFlow Configuration and Management Protocol), EMPP (extensible messaging and presence protocol), I2RS (interface to routing system), and so on. The most important OpenFlow protocol is a key protocol for the SDN network architecture developed by ONF.

The main function of the controller plane is to control the transmission of the data plane through the south interface, and to open the network to the application plane through the north interface. The SDON controller plane supports the connection control, network virtualization, centralization and the ability to provide third party applications in multi-domain, multi-technology, multi-level, and multi-vendor transport network.

The hierarchical control architecture of SDON controls the different network domains by the lower-level controller, and is responsible for inter domain collaboration through a higher-level controller. It realizes the logical centralized control architecture of the hierarchical domain. Interfaces between layers of controllers interact through I-CPI (intermediate-controller plane interface). The hierarchical control architecture has three basic patterns, as shown in Fig. $2 \mathrm{a}-\mathrm{c}$.
This paper is mainly based on Fig. $2 \mathrm{C}$ control architecture model.

\subsection{Two multi-domain control scheme and the hierarchical control system structure}

At present, there are two main ideas to realize multidomain control. The first scheme is adding west-east interface to connect controller on SDON control plane which realize the parallel data sharing and coordination among multiple SDON controllers [18-20]. This method still uses distributed control plane, so the realizing of cross-domain services still depends on more than one SDON controller. So that it is difficult to realize centralized control based on the whole network abstraction. The second scheme is using the soft-defined control structure of multi-domain optical network, in other words, using based on single-domain controller-multi-domain controller hierarchical control scheme. Each network domain distributed its own SDON controller, and it is called a single domain controller. All single domain controllers are connected with multi-domain controller through Openflow protocol. The multi-domain controller manages the abstraction information of the whole network, and it can centralize control cross domain service and global resource optimization procedure. This paper is based on the hierarchical control system in the second scheme.

Figure 3 illustrates the hierarchical control system structure based on single-domain controller-multi-domain controller. The system composed of four layers, the transmission equipment of the data plane, the single domain SDON controller in every network, multidomain controller, and application layer.

APP on application use HTTP (Hyper Text Transfer Protocol) protocol to communicate with multi- domain controller through A-CPI, the interface uses Restful style. All resource (such as node, port, link, etc.) in multidomain can be mapped to URL (Uniform Resource Locator). By combining network resources URL and HTTP protocol, A-CPI can cooperate with JSON (JavaScript Object Notation) information to achieve the application layer

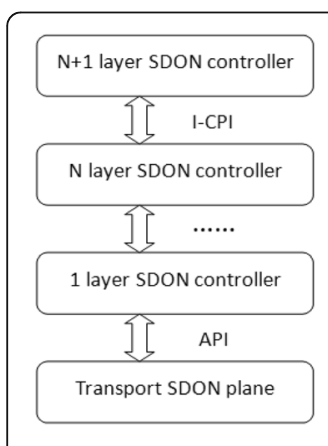

(a) $\mathrm{N}$ layer SDON controllers

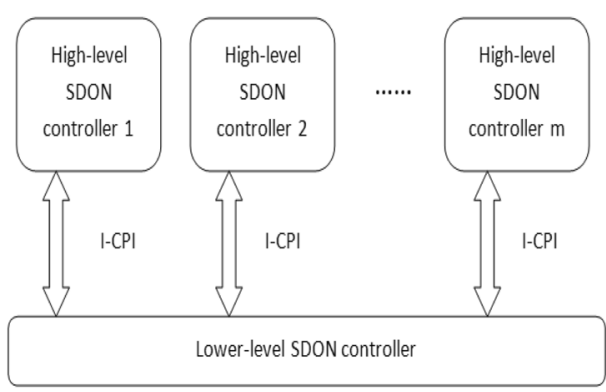

(b) One lower and N high-level SDON controllers

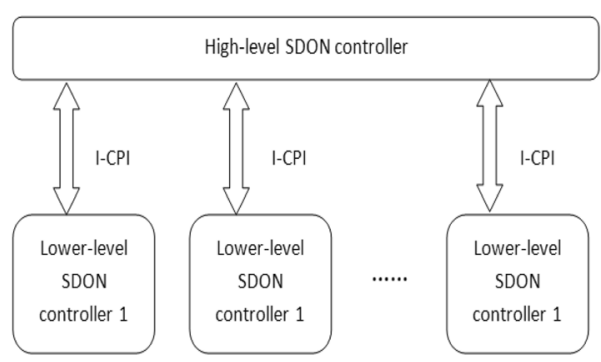

(c) N lower and one high-level SDON controllers

Fig. 2 The three basic patterns of the hierarchical control architecture 


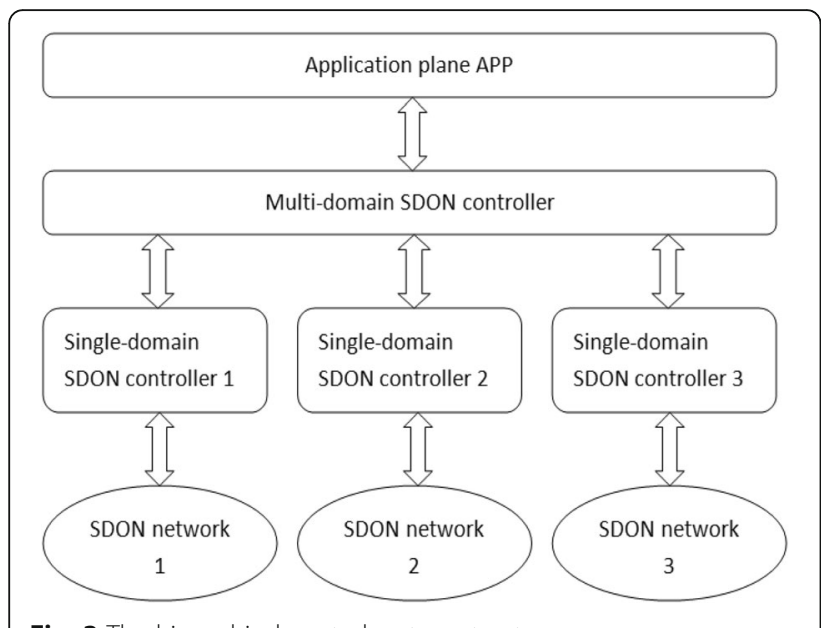

Fig. 3 The hierarchical control system structure

APP's access to the whole network and business order, so that APP can realize the control and business request of multiple domains by means of the controller. Each singledomain controller is responsible for the network topology detection, path computation, connection control, protection, and restoration. The single domain controllers transfer the topology and network resources and those are abstracted to the multi-domain SDON controller through the interlayer interface I-CPI. The multi-domain SDON controller is responsible for inter-domain topology detection, cross-domain path computation, cross-domain endto-end connection control, and protection recovery functions of multiple single-domain SDON controllers. The transport plane communicates with the single-domain SDON controller through D-CPI.

The advantage of this system is that the service problems that is limited to one network domain is assigned to the single-domain controller, and the cross-domain service problem will be solved by multi-domain controller. At the same time, the multi-domain controller can subdivide cross-domain service problem into multiple single-domain service problems and send them to each single-domain controllers and solve these problems. The multi-domain controller is only responsible for integrating the operation results of single domain controller.

\section{The resources optimization methods}

The inter-domain spectrum resources optimization methods of software-defined multi-domain optical network consist of two methods: the routing resource optimization method and the spectrum resources optimization method.

\section{The routing resource optimization method}

\subsection{Network model and symbol definition}

A multi-domain network is represented by $G(V, E)$ and includes $M$ network domains, which are represented as $G^{m}\left(V^{m}, E^{m}\right)$. Network domain $G^{m}\left(V^{m}, E^{m}\right)$ includes $\mid V^{m}=$ $\left\{v^{m}\right\} \mid$ nodes and $\left|E^{m}=\left\{e^{m}\right\}\right|$ inter-domain links. If there is an inter-domain link between the path node $v_{p}^{m}$ of the $m$ domain and the $q$ node $v_{q}^{n}$ of the $n$ domain, we can mark this link as $e_{p, q}^{m, n}$. The service request can be represented as $t_{p, q}^{m, n}$, it means that set the $p$ node of the $m$ domain as resource node, and set the $q$ node of then domain as sink node of service request.

In hierarchical multi-domain software-defined optical network, the multi-domain controller stores all topology information $G^{L}\left(V^{L}, E^{L}\right)$ of the whole network domains, it includes the inter-domain link resource information. This topology information will be reported by every single-domain controllers. In most cases, this topology information includes logical nodes, logical links, and resource conditions on links in the network, and does not contain information about private strategy, physical equipment details, and resource providing forms.

In the hierarchical control architecture of the softwaredefined multi-domain optical network, after receiving a cross-domain service request that requires an end-to-end all optical path, the multi-domain controller firstly will calculate the cross-domain service based on its own topological resource information, then send the result of precomputation to the single-domain controller through Flow_Mod messages, so as to complete the service building in each domains. This resource optimization algorithm flow is shown as Table 1.

\subsubsection{Simulation results and discussion}

For the topology of NSF (National Science Foundation) network, we compare two architectures: hierarchical control and single control, using the shortest path of $\mathrm{K}$ algorithm. The simulation results are shown as Fig. 4. It is clear that the hierarchical control architecture has lower bandwidth blocking probability than the single control architecture at the same traffic load. Moreover, the less traffic load, the lower the bandwidth blocking

Table 1 Resource optimization algorithm

for $t_{p, q}^{m, n}$ do
Invoking the $K$ algorithm in $G^{L}\left(V^{L}, E^{L}\right)$ and calculate the shortest path
from $v_{p}^{m}$ to $v_{q}^{n}$, then range the path according to its length: $P=\left\{p_{1}, p_{2}\right.$,
$\left.\ldots p_{k_{\prime}}\right\} ;$
for $p_{k} \in P$ do
In this path, take the input node and output node of every domain
regard as the source node and the sink node of a sub path in this
domain. Produce the business requirements of every domain, and
send them to the related controller;
if All domain return OK
Path creation successful, jump out;
else
Path creation fail, the loop go on;
end if
end for
end for




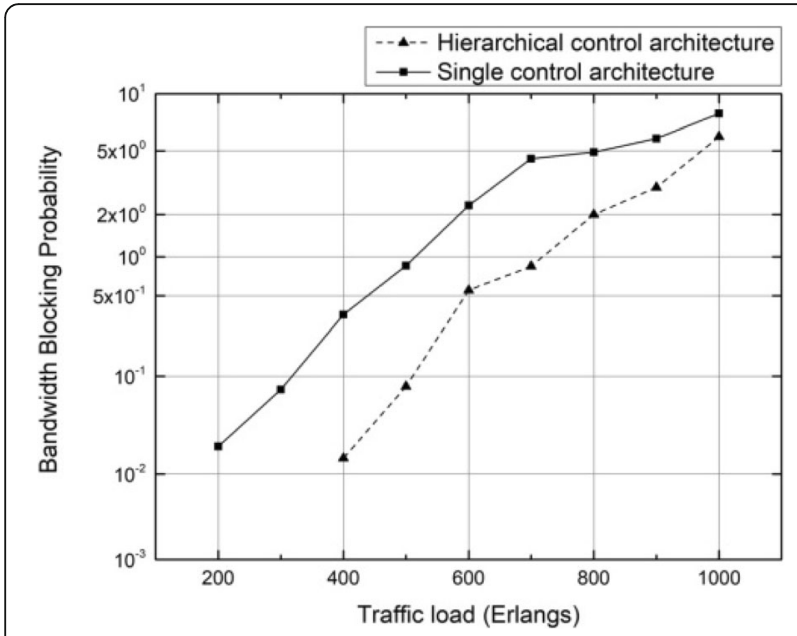

Fig. 4 The Bandwidth Blocking Probability under two architectures. The solid line and dotted line represent the bandwidth blocking probability of hierarchical control architecture and single control architecture, respectively

probability of the less of two architectures. The minimum is also above 0.02

\subsection{The spectrum resources optimization method}

\subsubsection{The traditional method of spectrum allocation}

When the optical paths span multiple domains, spectrum allocation needs to meet the following restrictions:

(1) Spectrum adjacency restriction: the allocated bandwidth slot is continuous on the spectrum dimension.

(2) Spectrum non-overlapping restriction: the bandwidth slot allocated to different requests cannot overlap each other, that is, a bandwidth slot cannot be occupied by more than one request at the same time.

(3) Spectrum continuity restriction: the allocated bandwidth slot is consistent on all links of the path.

At present, there are two kinds of traditional spectrum allocation methods: random distribution and first hit.

The random hit method searches for a path in its spectrum space and gives all available sets of spectral segments, and then randomly chooses a spectral bandwidth among all the available spectral segments to establish the optical route. As shown in Fig. 5a. The stochastic allocation of this method determines that it cannot improve the network performance well. It can easily cause congestion in the network without wavelength conversion.

In first hit method, all the available spectrum segments are labeled first. When searching for the available spectrum segments, low-numbered spectral segments are selected first, so that all the used spectral segments are as low as possible in the spectrum space. Therefore, there is a continuous, longer path of available spectrum at the high end of the spectrum space. As shown in Fig. $5 \mathrm{~b}$. The method does not need to traverse all the frequency gap resources and does not need to collect the running state of the network in real time. It is fast and simple, which is relatively easy to implement in practical applications.

\subsubsection{The problem of inter-domain spectrum allocation}

In a scenario in which multiple domains coexist, when a user initiates a cross-domain service request that requires possession of an end-to-end all-optical path, since it is not allowed to light/electricity/light wavelength conversion in the boundary node, the optical path across multiple domains also needs to meet the constraints of spectrum continuity and consistency. Therefore, the problem of spectrum fragments is particularly prominent. The dynamic access and random release of large capacity in the business domain cause the network spectrum resources to be fragmented after a period of time. These resource fragments cannot be reused, and new fragments are continuously generated, resulting in low utilization of network resources and high business blocking rate. Therefore, it is very important to study the optimal allocation of spectrum resources in multidomain scenarios, and spectrum fragments mechanism is one of the key technologies to optimize the allocation of spectrum resources.

In the case of large-scale and multi-domain optical networks, multi-domain controllers can have business and resource information of multiple domains at the same time. Therefore, an intelligent spectrum defragmentation mechanism based on actual network conditions can be implemented by using hierarchical control technology. In this paper, the spectrum defragmentation mechanism based on the degree of spectrum fragmentation is applied to the software-defined multi-domain optical network with hierarchical control architecture. And then compared with the software-defined multi-domain optical network in common architecture, this dissertation provides a better description of multi-domain light The Importance of Hierarchical Control Architecture in multi-domain optical networks.

\subsubsection{The arrangement of spectrum fragments}

The spectrum resource distribution in the link is described by using the spectrum resources required by the service, the actual spectrum resources occupied by the service, and the number of spectrum gaps. We can assume a link model as follows: there are $N$ services in a 


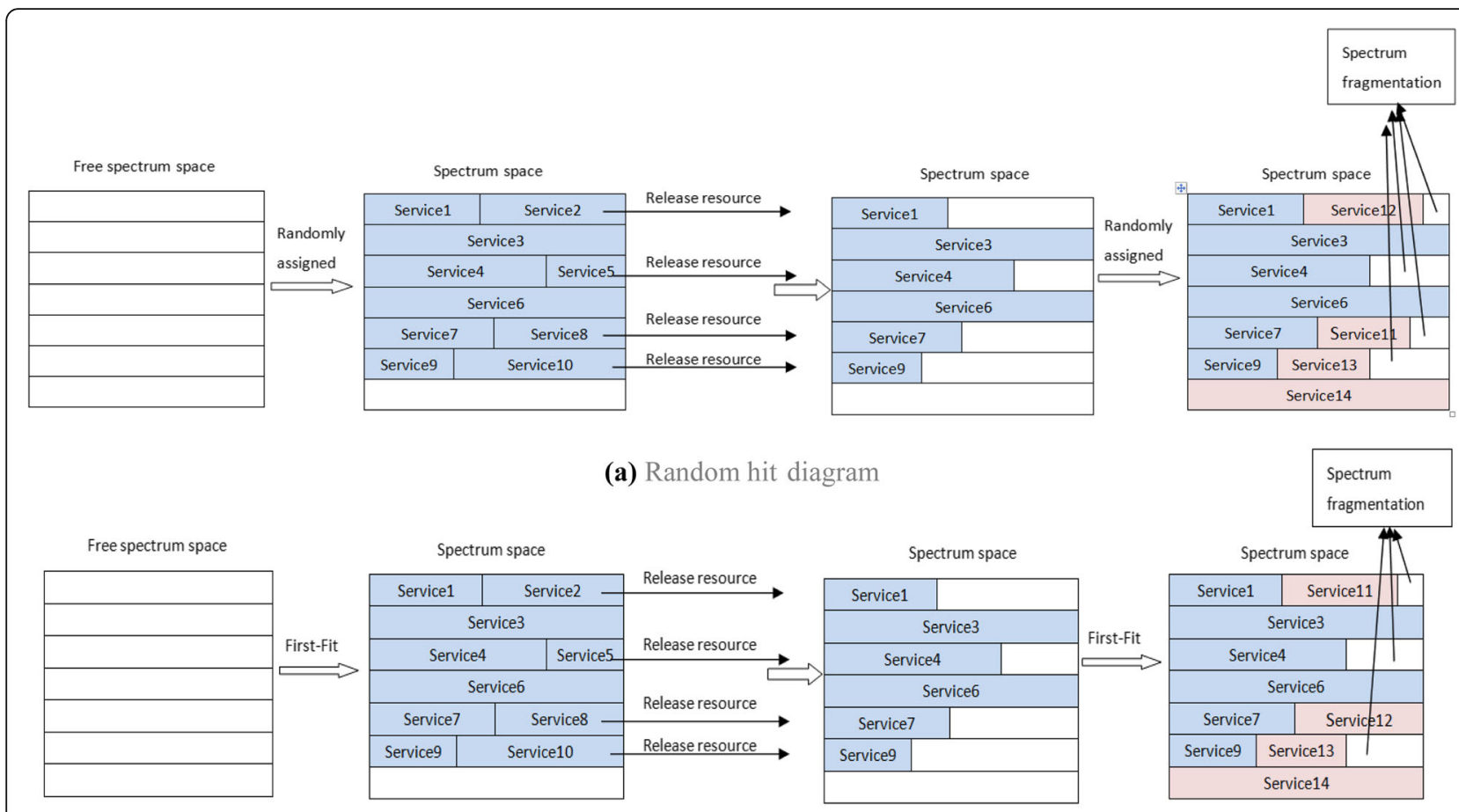

(b) First hitdiagram

Fig. 5 Two kinds of traditional spectrum allocation methods: random distribution and first hit

fiber link, and the spectrum resources used by the service are

$S_{i}(i=1,2, . . N)$, the lowest wavelength used by all services is $\lambda_{\min }$, the highest wavelength that has been used is $\lambda_{\max }$. Between $\left[\lambda_{\min }, \lambda_{\min }\right]$,the number of spectrum gaps is $k$, then the degree of spectral fragmentation of the link is

$$
F=\frac{\lambda_{\max }-\lambda_{\min }}{\sum_{i=1}^{N} S_{i}} * k
$$

Where, $\lambda_{\max }-\lambda_{\min }$ represents the spectrum resource occupied by the service in the link, $\sum_{i=1}^{N} S_{i}$ represents the spectrum resource required by the link, and $k$ represents the number of link gaps. The larger the $F$ value, the higher the spectrum fragmentation degree of the service, the higher the necessity of reconstruction.

The degree of link spectrum fragmentation is generalized to the entire software-defined multi-domain optical network. That is, using the degree of network spectrum fragmentation to count the entire optical network spectrum resource distribution status. The relevant network model for the degree of fragmentation of the network spectrum is as follows: given an optical network topology $G(V, E), \mathrm{V}$ represent nodes and $E$ represent fiber links of connecting nodes. It is assumed that there are already $N$ services in the network, where the $i$ th service uses the spectrum resource as $S_{i}$, the number of hops passed is $T_{i}\left(T_{i}<M, i=1,2, . . N\right)$. For a certain link $j$ $(j=1,2, . . M, M$ represents the total number of links in the optical network). The minimum wavelength that a service can use is $\lambda_{\min }^{j}$, the highest wavelength the service has used is $\lambda_{\max }^{j}$. Between $\left[\lambda_{\min }^{j}, \lambda_{\max }^{j}\right]$,the number of spectral gaps is $K_{j}$, then the degree of spectral fragmentation of the network is

$$
F=\frac{\sum_{j=1}^{M}\left(\lambda_{\max }^{j}-\lambda_{\min }^{j}\right)}{\sum_{i=1}^{N} S_{i} * T_{i}} * \frac{\sum_{j=1}^{M} K_{j}}{M}
$$

The larger the $F$ value, the higher the degree of fragmentation of the spectrum of the service is and the higher the necessity of reconstructing it; and vice versa.

Where $\frac{\sum_{j=1}^{M}\left(\lambda_{\max }^{j}-\lambda_{\min }^{j}\right)}{\sum_{i=1}^{N} S_{i} * T_{i}}$ represents the ratio of the total network spectrum resource occupied by the whole network service to the spectrum resource required by the 
$\sum_{j=1}^{M} K_{j}$

whole network service, and $\frac{j=1}{M}$ represents the average number of gaps on each link. By setting a threshold $T$ of the degree of spectral fragmentation and an excessively fragmented link ratio range $r$, delineating a set of optical links that need to be sorted. The network fragmentation value $F$ is calculated by Eq. (2), and if the $F$ is greater than the threshold $T$, the spectrum defragmentation mechanism is triggered.

The multi-domain controller is responsible for the calculation and monitoring of the value of the cross domain link, and adopts a separate monitoring mechanism and threshold for spectrum fragmentation of the cross domain link. Once the cross domain link $F$ value exceeds the threshold, the spectrum defragmentation of the cross domain service is triggered directly. To differentiate intra-domain services from inter-domain services, OpenFlow messages need to be extended to interact with the service ID list between the multi-domain controller and the single-domain controller. The Reason field of the Packet in message distinguishes between single-domain and multi-domain upload (or multidomain) delivery to a single-domain.

\subsubsection{Simulation results and discussion}

The simulation results are based on the NSF network topology. There are 14 network nodes and 21 links. The optical fiber is bi-directional. Every link has $1 \mathrm{~T}$ spectrum resource. The business arrival rate is the Poisson distribution, and business service rate is negative exponential distribution. The bandwidth required for each business obeys the uniform distribution. Setting the threshold of spectrum fragmentation degree $T=200$, the simulation of the bandwidth blocking probability and the spectrum efficiency is shown as Figs. 6 and 7, respectively.

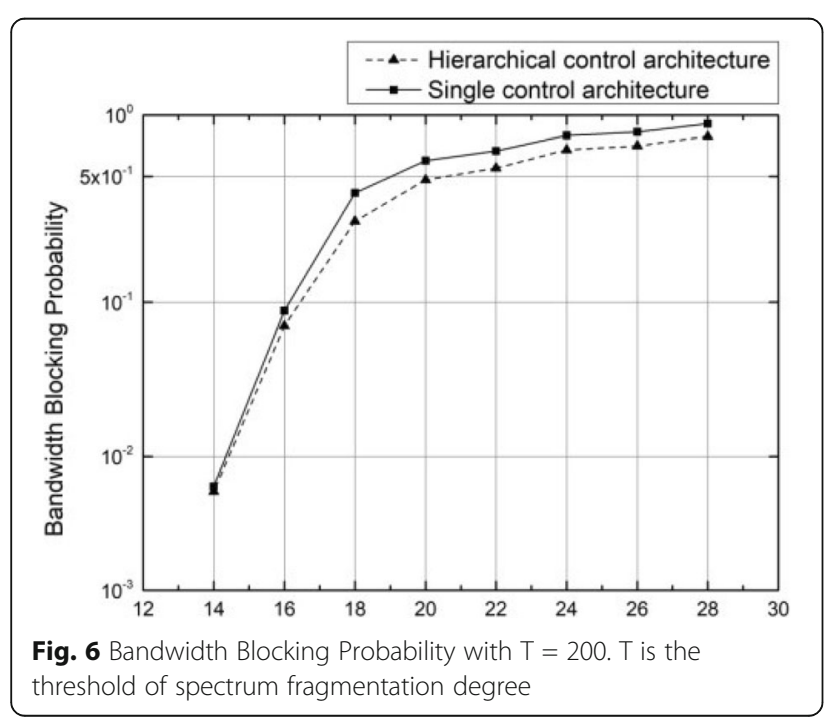

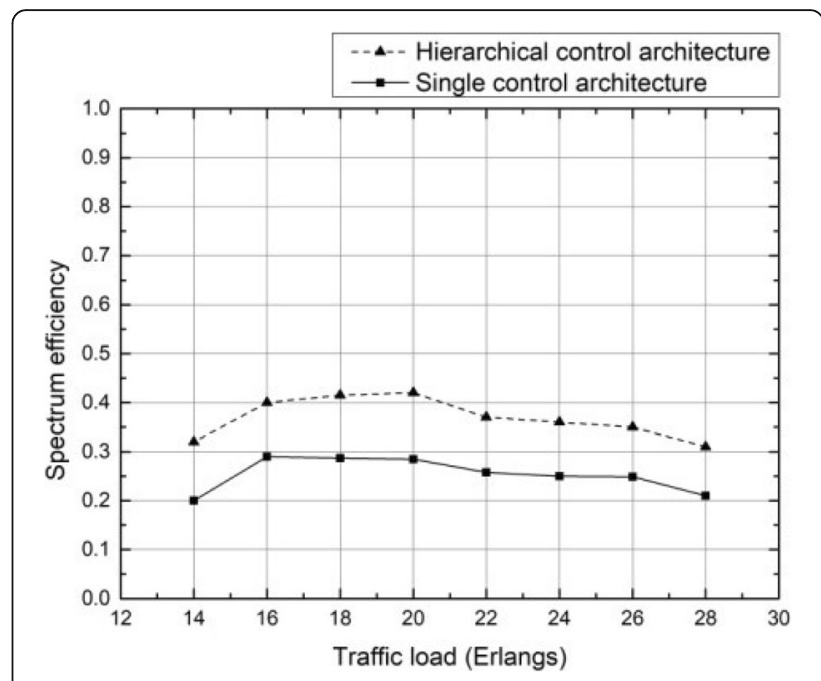

Fig. 7 Spectrum efficiency with $T=200$. $T$ is the threshold of spectrum fragmentation degree

Setting the threshold of spectrum fragmentation degree $T=100$, the simulation of the bandwidth blocking probability and the spectrum efficiency is shown as Figs. 8 and 9 , respectively.

The simulation results show that when the threshold of spectrum fragmentation degree $T$ is lower, the spectrum efficiency is higher, but the bandwidth blocking probability is almost constant at the corresponding traffic load. For the two kinds of control architecture, the hierarchical control architecture has lower bandwidth blocking probability and higher spectrum efficiency than the single control architecture at the same traffic load. When setting the threshold of spectrum fragmentation degree $T=100$, the spectrum efficiency of the hierarchical control architecture is up to more than $90 \%$. The results mean the hierarchical

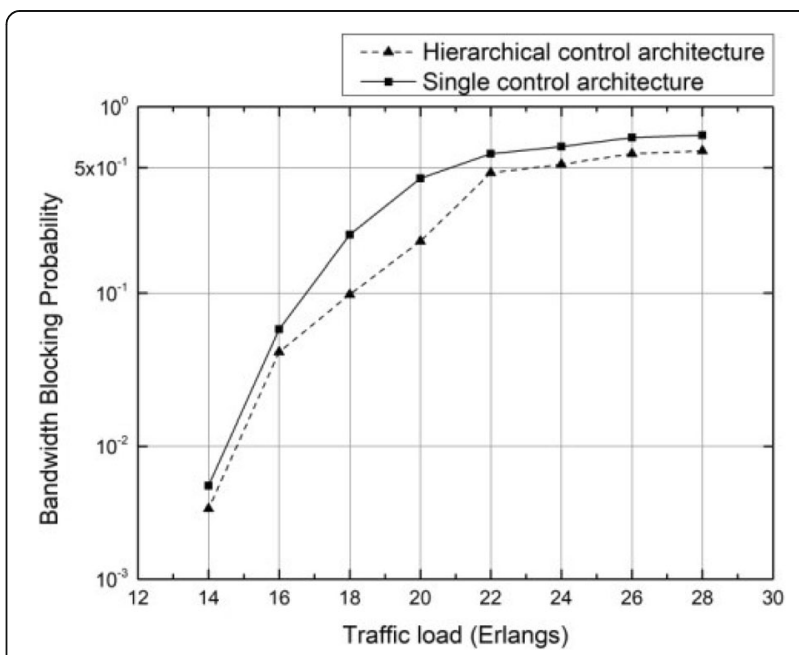

Fig. 8 Bandwidth Blocking Probability with $T=100$. $T$ is the threshold of spectrum fragmentation degree 


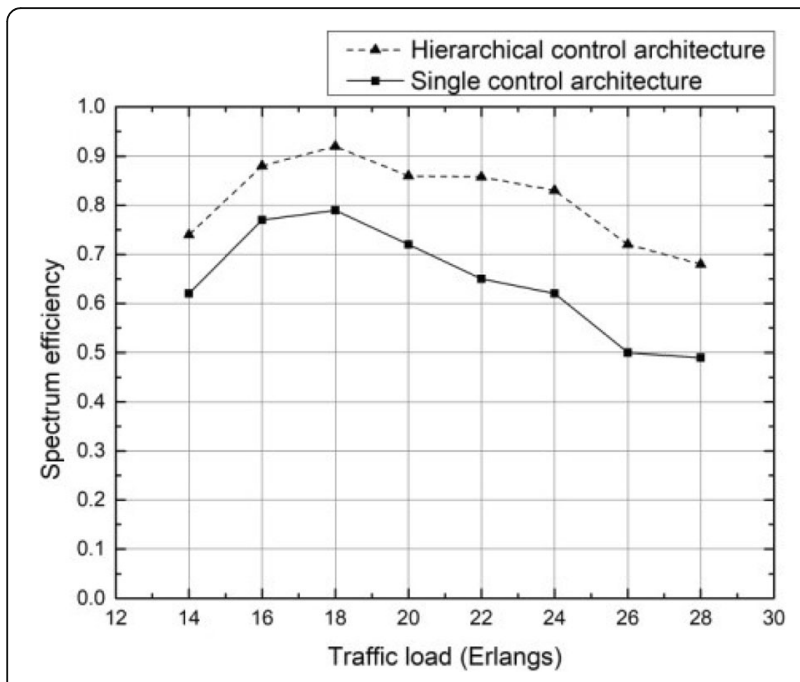

Fig. 9 Spectrum efficiency with $T=100$. $T$ is the threshold of spectrum fragmentation degree

control effectively reduces the network blocking rate and improve the spectrum efficiency. It indicates that the superiority of hierarchical control system.

\section{Conclusions}

In this paper, the multi-level control architecture is applied to the architecture of software-defined optical network, aiming at the problem of resource allocation among domains in the multi-domain optical network scenario. The coordination of the single-domain controller and the multi-domain controller control the domain resource scheduling in the multi-domain optical network. The single control architecture and hierarchical control architecture of resource optimization algorithms are simulated and analyzed. The result shows that the hierarchical control system does reduce the computational complexity of the controller, so that the traffic congestion rate is reduced and the spectrum utilization rate is obviously enhanced. But this paper only uses the simplest $K$ shortest path algorithm and stochastic routing algorithm, so for some of the routing spectrum configuration algorithm has been proposed, we also need further research and experimental simulation to propose better inter-domain resource optimization algorithm.

\footnotetext{
Abbreviations

A-CPI: Application plane control interface; API: Application programming interface; APP: Application; ASON: Automatic Switching Optical Network; CPI: Intermediate-controller plane interface; D-CPI: Data plane control interface; EMPP: Extensible messaging and presence protocol; HTTP: Hyper Text Transfer Protocol; I2RS: Interface to routing system; I-CPI: Intermediatecontroller plane interface; JSON: JavaScript Object Notation; NSF: National Science Foundation; OFCONFIG: OpenFlow Configuration and Management Protocol; ONF: Open Networking Foundation; O-OFDM: Orthogonal frequency division multiplexing; PCE: Path computation element; SDH: Synchronous digital hierarchy; SDN: Software-defined network; SDON: Defined optical network; SNMP: Simple Network Management
}

Protocol; SOAP: Simple Object Access Protocols; URL: Uniform Resource Locator; WDM: Wavelength-division multiplexing

\section{Acknowledgements \\ Not applicable.}

\section{Authors' contributions}

$\mathrm{ZL}$ and $\mathrm{YC}$ finished the algorithm and English writing of the paper. SH finished the experiments. ZZ put forward the idea of this paper. All authors read and approved the final manuscript.

\section{Funding}

This work was supported by the National Natural Science Foundation of China under grant 61661004 and Guangxi Science Foundation under grant 2017GXNSFAA198227.

\section{Availability of data and materials}

Data sharing is not applicable to this article as no datasets were generated or analyzed during the current study

\section{Competing interests}

The authors declare that they have no competing interests.

Received: 25 October 2019 Accepted: 3 February 2020

Published online: 13 February 2020

\section{References}

1. Y. Sun, G. Feng, S. Qin, T.-S.P. Yum, Y.-C. Liang, The SMART handoff policy for millimeter wave heterogeneous cellular networks. IEEE Trans. Mobile Comput. 17(6), 1456-1468 (2018)

2. Y. Sun, G. Feng, S. Qin, S. Sun, Cell association with user behavior awareness in heterogeneous cellular networks. IEEE Trans. Vehicular Technol. 67(5), 4589-4601 (2018)

3. Y. Sun, L. Zhang, G. Feng, B. Yang, B. Cao, M.A. Imran, Blockchain-enabled Wireless Internet of Things: Performance Analysis and Optimal Communication Node Deployment. IEEE Internet Things J. 6(3), 5791-5802 (2019)

4. Y.Y. Zhenrong Zhang, F. Songnian, Broadband On-Chip mode-division multiplexer based on adiabatic couplers and symmetric Y-Junction. IEEE Photonics J. 9(2) (2017)

5. Z. Zhang, M. Xiao, M. Wu, F. Xie, Adaptive subcarrier-distribution algorithm for routing and spectrum allocation in OFDM-based elastic optical networks. Photon Netw. Commun. 28(3), 225-231 (2014)

6. Yu Zhou, Shanguo Huang, Wenzhe Li, et al, Towards software-defined optical network: from the perspective of heterogeneous optical networks. Paper presented at 2015 Opto-Electronics and Communications Conference, Shanghai, China, 28 June-2 July 2015.

7. L. Yang, J. Luo, Y. Xu, Z. Zhang, Z. Dong, A Distributed dual consensus ADMM based on partition for DC-DOPF with carbon emission trading. IEEE Trans. Industrial Inform. (2019). https://doi.org/10.1109/TII.2019.2937513

8. L. Yang, C. Zhang, J. Jian, K. Meng, Y. Xu, Z. Dong, A novel projected twobinary-variable formulation for unit commitment in power systems. Appl. Energy 187, 732-745 (2017)

9. N. Mckeown, T. Anderson, H. Balakrishnan, et al., OpenFlow: enabling innovation in campus networks. AcmSigcomm Comp. Commun. Rev. 38(2), 69-74 (2008)

10. M. Channegowda, R. Nejabati, D. Simeonidou, Software-defined optical networks technology and infrastructure: enabling software-defined optical network operations [Invited]. J. Optical Commun. Networking 5(10), A274 (2013)

11. Philip N. Ji, Software defined optical network. Paper presented at the 2012 11th International Conference on Optical Communications and Networks, Chonburi, Thailand, 28-30 Nov 2012.

12. Chen M, Shou G, Hu Y, et al, Enabling software-defined optical networks based on OpenFlow extension. Paper presented at 2015 Opto-Electronics and Communications Conference, Shanghai, China, 28 June-2 July 2015.

13. Y. Ji, J. Zhang, Y. Zhao, Development prospects of software defined optical networks. Telecommunications Science 30(8), 19-22 (2014)

14. Yiming Yu, Yi Lin, Jie Zhang, et al. Field demonstration of datacenter resource migration via multi-domain software defined transport networks 
with multi-controller collaboration. Paper presented at Optical Fiber Communication (OFC) Conference, San Francisco, CA, USA, 28 August 2014.

15. X. Liu, R. Gu, H. Li, Y. Tan, L. Wang, et al, Demonstration of Hierarchical Control for Multi-domain and Multi-vendor Software-defined Packet

Transport Network. Paper presented at Asia Communications and Photonics Conference, Hong Kong, China, 19-23 November 2015.

16. Zhang J W, Zhao Y L, Ji Y F, Software definition of optical network technology evolution and innovation application. Information and Communication Technology, Lett.1, 10-16(2016).

17. A.A. Bruno, M.M. Nunes, X.-N. Nguyen, et al., A Survey of software-defined networking: past, present, and future of programmable networks. IEEE Commun. Surveys Tutorials 16(3), 1617-1634 (2014)

18. Fei, Song, and H. Yueqing, Challenges and Development Trend of SDN Technology. Information and Communications Technologies (2015).

19. C. Xue, C.Q. Ma, Z.B. Liu, et al., A security SDN controller architecture design. Information and network Security Lett 9, 34-38 (2014)

20. F. Yang, C. Li, T. Huang, OXP: An east-west protocol for SDN mobile ad-hoc networks. Telecom Engineering Technics Standardization 29(9), 32-37 (2016)

\section{Publisher's Note}

Springer Nature remains neutral with regard to jurisdictional claims in published maps and institutional affiliations.

\section{Submit your manuscript to a SpringerOpen ${ }^{\circ}$ journal and benefit from:}

- Convenient online submission

- Rigorous peer review

- Open access: articles freely available online

- High visibility within the field

- Retaining the copyright to your article

Submit your next manuscript at $\boldsymbol{\nabla}$ springeropen.com 